International Journal of Modern Physics D

(C) World Scientific Publishing Company

\title{
Disentangling birefringence from standard physics in CMB measurements and distinguishing among production mechanisms
}

\author{
GIULIA GUBITOSI
}

Theoretical Physics, Blackett Laboratory, Imperial College, London, SW7 2BZ, United Kingdom g.gubitosi@imperial.ac.uk

Received Day Month Year Revised Day Month Year

\begin{abstract}
The search for possible effects of parity-violations in the electromagnetic sector has achieved great accuracy with the latest Cosmic Microwave Background (CMB) observations. The sensitivity of current and future experiments is such that the need to understand the interaction of parity violations with several competing effects is now crucial in order to improve current constraining power. It also allows to not only put constraints on the most generic features of parity violations, but also for distinguishing between several production mechanisms, if any effect is ever observed. The results here reported were presented at the Cosmic Polarization Rotation workshop held in Florence on the 7th and 8th of September, 2015.
\end{abstract}

Keywords: Cosmic Microwave Background Polarization; Birefringence; Cosmic Polarization Rotation; Parity Violation

PACS numbers:

\section{Introduction}

In the past years attempts to detect the effects of parity-violating physics in the Cosmic Microwave Background (CMB) polarization have multiplied. ${ }^{1-14}$ The main signal of parity-violation would be a correlation between parity-even (polarization E modes and temperature T modes) and parity-odd (polarization B modes) components of the CMB anisotropies. The power gained by the parity-violating correlation power spectra would result in a loss of power from the other ones (EE, TE, BB correlations). More specifically, if the mixing happened in a sudden way at the detection of the CMB radiation, the observed power spectra, $\mathcal{C}_{\ell}$, would be related to the non parity-violating ones, $\tilde{C}_{\ell}$, by:

$$
\begin{aligned}
C_{\ell}^{E E} & =\tilde{C}_{\ell}^{E E} \cos ^{2}(2 \beta)+\tilde{C}_{\ell}^{B B} \sin ^{2}(2 \beta)-\tilde{C}_{\ell}^{E B} \sin (4 \beta) \\
C_{\ell}^{B B} & =\tilde{C}_{\ell}^{E E} \sin ^{2}(2 \beta)+\tilde{C}_{\ell}^{B B} \cos ^{2}(2 \beta)+\tilde{C}_{\ell}^{E B} \sin (4 \beta) \\
C_{\ell}^{E B} & =\frac{1}{2}\left(\tilde{C}_{\ell}^{E E}-\tilde{C}_{\ell}^{B B}\right) \sin (4 \beta)+\tilde{C}_{\ell}^{E B}\left(\cos ^{2}(2 \beta)-\sin ^{2}(2 \beta)\right) \\
C_{\ell}^{T E} & =\tilde{C}_{\ell}^{T E} \cos (2 \beta)-\tilde{C}_{\ell}^{T B} \sin (2 \beta) \\
C_{\ell}^{T B} & =\tilde{C}_{\ell}^{T E} \sin (2 \beta)+\tilde{C}_{\ell}^{T B} \cos (2 \beta),
\end{aligned}
$$


where $\beta$ is a dimensionless parameter, usually measured in degrees, giving the amount of parity violation ${ }^{a}$. The kind of mixing described by these equations is the one generated by a rotation of the polarization plane of radiation when radiation is linearly polarized. In this case the parameter $\beta$ indicates the amount of rotation. This phenomenon is know as in-vacuo birefringence, or cosmic polarization rotation (note that in ${ }^{15,16}$ it is suggested that a distinction should be made between in-vacuo birefringence and cosmic polarization rotation, the last one being characterized by energy-independence and the former by a more general behaviour, such as energy and direction dependence). Nowadays the ever increasing precision of CMB experiments allows in principle to constrain $\beta$ to less than a degree, or even one order of magnitude smaller. ${ }^{17-19}$ However, several competing effects could be affecting our ability of detecting the sort of mixing described by eqs.(2). These range from systematic errors, due to miscalibration of the polarimeters in the detectors, to the effects of gravitational lensing and the B modes generated by primordial tensor modes. And, vice-versa, if the parity violation effect were indeed present, it could limit our ability to properly measure standard-physics parameters if not properly taken into account ${ }^{\mathrm{b}}$. These topics will be the focus of the following section 2 . In section 3 we are going to discuss how to discern among possible parity-violation mechanisms if any effect is seen.

\section{Parity violation vs. standard physics effects}

We have stated already that the characteristic feature of parity-violation in the CMB is a mixing between $E$ and $B$ polarization modes. A similar transfer of power in the polarization is produced by weak lensing. Even though lensing itself does not generate correlations between $\mathrm{E}$ and $\mathrm{B}$ modes, it transfers power between $E E$ and $B B$ power spectra. Moreover, if some parity-violation mechanism is at play, weak lensing further enhances the $E B$ and $T B$ cross-correlations. In fact, given some initial power spectra $C_{\ell}$, the lensed real-space correlation functions, depending on the angle $\gamma$ between two observation directions, are: ${ }^{21}$

$$
\begin{aligned}
& \bar{\xi}_{X}(\gamma)=\sum_{\ell} \frac{2 \ell+1}{4 \pi}\left(C_{\ell}^{T E}-i C_{\ell}^{T B}\right) F_{X}^{\ell} \\
& \bar{\xi}_{+}(\gamma)=\sum_{\ell} \frac{2 \ell+1}{4 \pi}\left(C_{\ell}^{E E}+C_{\ell}^{B B}\right) F_{+}^{\ell} \\
& \bar{\xi}_{-}(\gamma)=\sum_{\ell} \frac{2 \ell+1}{4 \pi}\left(C_{\ell}^{E E}-C_{\ell}^{B B}-2 i C_{\ell}^{E B}\right) F_{-}^{\ell},
\end{aligned}
$$

\footnotetext{
$\overline{{ }^{a} \text { Of course the power spectra } \tilde{C}_{\ell}^{T B} \text { and }} \tilde{C}_{\ell}^{E B}$ are zero in absence of additional parity violations at emission or during propagation of the CMB.

${ }^{\mathrm{b}}$ There are other proposed physical effects which are not pariy-violating and still would generate a rotation of polarization. For example $i^{20}$ an effective interaction in terms of a CPT-even dimension-six term is considered. $\operatorname{In}^{16}$ some other examples of non-parity-violating Lagrangians are discussed. Even tough these models are of interest, here we are going to focus on parity-violating models.
} 
where $F_{X,+,-}^{\ell}$ are functions containing geometrical factors and the the lensing potential. ${ }^{21}$ From these correlation functions one can recover the lensed power spectra using:

$$
\begin{aligned}
\bar{C}_{\ell}^{T E}-i \bar{C}_{\ell}^{T B} & =2 \pi \int_{-1}^{1} \bar{\xi}_{X} d_{20}^{\ell} d \cos \gamma \\
\bar{C}_{\ell}^{E E}+\bar{C}_{\ell}^{B B} & =2 \pi \int_{-1}^{1} \bar{\xi}_{+} d_{22}^{\ell} d \cos \gamma \\
\bar{C}_{\ell}^{E E}-\bar{C}_{\ell}^{B B}-2 i \bar{C}_{\ell}^{E B} & =2 \pi \int_{-1}^{1} \bar{\xi}_{-} d_{2-2}^{\ell} d \cos \gamma,
\end{aligned}
$$

where $d_{s s^{\prime}}^{\ell}(\gamma) \equiv \sum_{m}{ }_{s} Y_{\ell m}^{*}\left(\hat{n}_{1}\right)_{s^{\prime}} Y_{\ell m}\left(\hat{n}_{2}\right), \hat{n}_{1}$ and $\hat{n}_{2}$ being the observation directions. If no parity-violating effect is present, one can set the $C_{\ell}^{T B}$ and $C_{\ell}^{E B}$ to zero in eq. (2) and no $\bar{C}_{\ell}^{T B}$ nor $\bar{C}_{\ell}^{E B}$ will be generated in eq. (3). The $E B$ and $T B$ correlations might in this case only be generated by a systematic error, such as a miscalibration of the polarimeters. ${ }^{22}$ The miscalibration would act as in eq. (2), where the $\tilde{C}_{\ell}$ would be the lensed spectra (and so $\tilde{C}_{\ell}^{E B}$ and $\tilde{C}_{\ell}^{T B}$ would be zero). However, since weak lensing is a late-time phenomenon, if the parity violation is present and due to an effect that accumulates over the full propagation history of $\mathrm{CMB}$ radiation, it is to be expected that weak lensing will act on spectra that already have non-zero $E B$ and $T B$ correlations (in this case we substitute the parameter $\alpha_{0}$ to $\beta$ to quantify the amount of mixing in eq. (2)). Thus there will be a mixing between $E E, B B$ and $E B$ power spectra as well as between $T E$ and $T B$, following eq. (3). In Fig. 1 we compare the power spectra resulting from several different cases: standard $\Lambda \mathrm{CDM}$ including lensing, standard $\Lambda \mathrm{CDM}$, including lensing, with a systematic error (i.e. the parity-violating-like mixing of the spectra happens at the detection), $\Lambda \mathrm{CDM}$ with time-independent parity violation effect and with time evolving parity violation (see following section for an explanation of this last option). The main difference between the systematic error case and the constant parity-violating case resides in the low- $\ell B B$ and $E B$ cross-correlation power spectra.

In a previous work ${ }^{21}$ constraints on both the systematic error and the parity violation effect were derived using WMAP9 and BICEP1 data. These datasets were shown not to be sensitive enough to distinguish between the two effects (see Table 1 ), even though they favour some non-zero effect (be it systematic error or genuine parity-violating physics). On the other hand, it was also shown ${ }^{21}$ that more recent datasets, such as Planck and PolarBear, would have the sensitivity to distinguish between the two options. And moreover, if the amount of effect were the one favoured by WMAP9+BICEP1 data, the new datasets would provide a detection (see Table 2).

Besides the interaction between the mixing of modes due to lensing and the one due to systematic or parity-violating effects, one should also worry about the consequences of neglecting any other mixing besides the one due to lensing. In 
this case, if parity violation or systematic errors were indeed present, by neglecting them one would risk to have a false detection of non-standard lensing amplitude, ${ }^{21}$ $A_{L}>1$. A non-standard $A_{L}$ can in fact compensate for the bias on the cosmological parameters induced by neglecting the parity-violation or systematic error. This is shown by analyzing a mock Planck+PolarBear dataset which originally includes a parity-violating effect $\alpha_{0}$ (fiducial model given by first column of Table 1). Results are in Table 3. Note that if no parity-violating effect is assumed, one gets a wrong estimate of the cosmological parameters (second column of the table). In order to compensate for the bias induced by a parity-violating effect as small as $\alpha_{0}=$ $(2.3 \pm 0.008) \cdot 10^{-2}$ one needs $A_{L}=1.29 \pm 0.03$ (third column of the table), i.e. a false detection of non-standard lensing amplitude.

Another false detection that may be caused by disregarding the effects of parity violations or polarimeters miscalibrations concerns primordial gravitational waves. ${ }^{22}$ The leak of power to BB modes described by eq. (2) could in fact mimic the presence of tensor modes. As shown in Table 4, the tensor-to-scalar ratio upper limit for Planck is affected by neglecting a mixing angle $\beta$ of order $1^{\circ}$. With higher-precision experiments, such as EPIC, one would have false detection of $r$ already for $\beta \sim$ $0.5^{\circ} \cdot{ }^{22}$
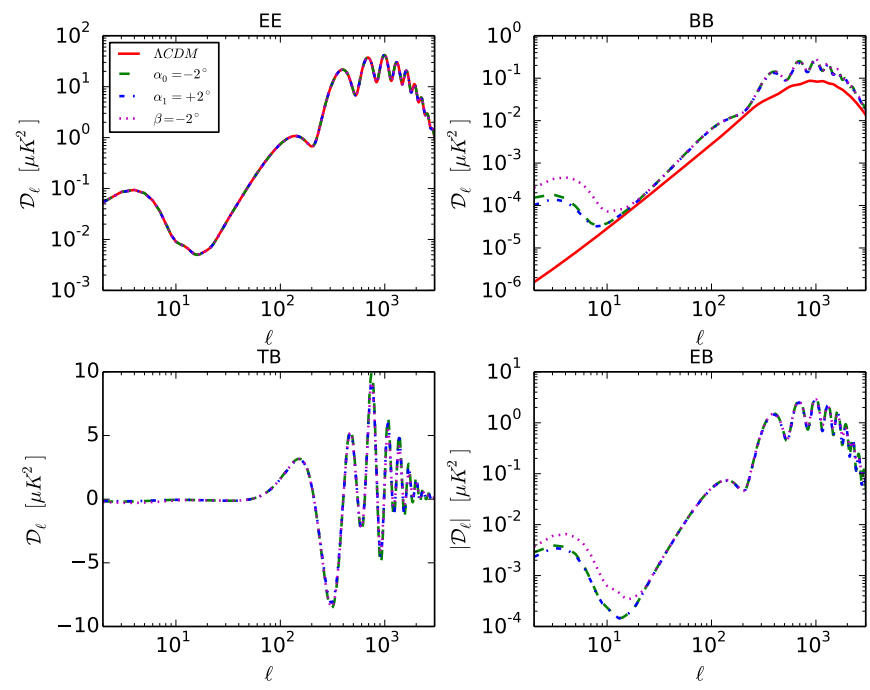

Fig. 1. $\quad \mathrm{CMB}$ power spectra obtained in standard $\Lambda \mathrm{CDM}$ (red solid line) including lensing, standard $\Lambda$ CDM including lensing with a systematic parity-violating-like effect (pink dotted line) $\Lambda C D M$ with time-independent parity violation effect (green dashed line) and with time evolving parity violation (blue dashed line). ${ }^{21}$ Note that there is no red line in the TB and EB panels as these cross-correlation power spectra are zero in the standard $\Lambda$ CDM scenario. 


\section{Distinguishing different parity-violating mechanisms}

If any parity-violating effect is seen in the data, the issue of distinguishing between different production mechanisms will become crucial (a non-exhaustive list of production mechanisms can be found $\mathrm{in}^{23}$ ). The main distinguishing features of different mechanisms are the dependence on the CMB radiation energy and the time evolution of the effect during propagation of $\mathrm{CMB}^{21,23,24 \mathrm{c}}$. A detailed analysis of how to constrain the time evolution of the effect was reported in a previous work by the author and collaborators, ${ }^{21}$ here we will focus on the energy dependence. In particular, the known models for parity-violations either have and energy dependence which is linear, or quadratic, or inverse quadratic or they are energy-independent (this last case is the one that some authors ${ }^{15,16}$ suggest calling cosmic polarization rotation). These possibilities can be formalized as:

$$
\begin{aligned}
& \beta(E)=q E \\
& \beta(E)=l E^{2} \\
& \beta(E)=\beta_{0} \\
& \beta(E)=h E^{-2}
\end{aligned}
$$

The energy-dependence of parity-violating mechanisms can be studied by comparing constraints on parity-violation coming from different CMB experiments, as they measure the CMB radiation at slightly different frequencies. In Table 5 we report the constraints on the parity-violating parameter $\beta$ coming from several experiments, along with the frequency at which the measurements are done. These data were analyzed with respect to the energy dependence ${ }^{23}$ and lead to the results reported in Table 6. One can see that if the QUAD $150 \mathrm{GHz}$ channel is included in the analysis then all the parameters for the possible energy dependence models are within one standard deviation from zero. However, if the QUAD $150 \mathrm{GHz}$ channel is excluded from the analysis (and this would be justifiable because several doubts have been independently raised about its reliability, $\mathrm{see}^{23}$ and references therein), all but the inverse-quadratic-dependence parameters are $\sim 2.5$ standard deviations away from zero. It is not possible to single out a favoured model among the remaining three because of too big experimental errors, however this would change were more precise data to be used, such as the ones of Planck. Indeed, in this case one could separately analyze the data of the several Planck channels and would actually be able to discriminate among the models as sensitivity on all the parameters would improve by one order of magnitude. ${ }^{23}$

\section{Conclusions}

The experimental sensitivity reached by CMB observations allows to put stringent constraints on parity-violations in the electromagnetic sector as well as on several

${ }^{\mathrm{c}}$ Also the (non-)isotropy of the effect can be used to discriminate between models, however this is still a under-developed point (see however ${ }^{25,26}$ ). 
standard physics effects, such as weak lensing and presence of primordial tensor modes. All these have similar signatures in the CMB, so it is necessary to develop techniques to properly distinguish among them. The same techniques can be also useful in order to separate the effects of systematic errors due to miscalibration of polarimeters from actual physical effects (be them due to either standard or non-standard physics). If any parity-violation effect is actually found, the study of the energy dependence of the effect can be used to distinguish among possible production mechanisms.

\section{Acknowledgments}

The author is supported by a grant from the John Templeton Foundation. The author wishes to thank the organizers of the Cosmic Polarization Rotation Workshop for the hospitality in such an inspiring location and for creating this opportunity for fruitful exchange of ideas among the participants. The author also wishes to thank M. Martinelli, F. Paci and L. Pagano, who were involved in a big part of the work that she presented at the workshop.

\section{References}

1. Kaufman, J. P., Miller, N. J., Shimon, M., et al. 2014, Phys. Rev. D, 89, 062006

2. A. Lue, L. M. Wang and M. Kamionkowski, Phys. Rev. Lett. 83, 1506 (1999) [arXiv:astro-ph/9812088].

3. N. F. Lepora, arXiv:gr-qc/9812077.

4. V. A. Kostelecky and M. Mewes, Phys. Rev. D 66 (2002) 056005 [arXiv:hep$\mathrm{ph} / 0205211]$.

5. J. Q. Xia, H. Li, X. l. Wang and X. m. Zhang, Astron. Astrophys. 483 (2008) 715 [arXiv:0710.3325 [hep-ph]].

6. F. Finelli and M. Galaverni, Phys. Rev. D 79 (2009) 063002 [arXiv:0802.4210 [astro$\mathrm{ph}]$.

7. T. Kahniashvili, R. Durrer and Y. Maravin, Phys. Rev. D 78 (2008) 123009 [arXiv:0807.2593 [astro-ph]].

8. G. Gubitosi, L. Pagano, G. Amelino-Camelia, A. Melchiorri and A. Cooray, arXiv:0904.3201 [astro-ph.CO].

9. P. Cabella, P. Natoli \& J. Silk, 2007, prd, 76, 123014

10. K. R. S. Balaji, R. H. Brandenberger and D. A. Easson, JCAP 0312, 008 (2003) [arXiv:hep-ph/0310368].

11. A. Gruppuso, P. Natoli, N. Mandolesi, A. De Rosa, F. Finelli and F. Paci, JCAP 1202 (2012) 023 [arXiv:1107.5548 [astro-ph.CO]].

12. G. Gubitosi and F. Paci, JCAP 1302 (2013) 020 [arXiv:1211.3321 [astro-ph.CO]].

13. M. Giovannini and K. E. Kunze, Phys. Rev. D 79 (2009) 087301 [arXiv:0812.2804 [astro-ph]].

14. A. P. S. Yadav, M. Shimon and B. G. Keating, Phys. Rev. D 86 (2012) 083002 [arXiv:1207.6640 [astro-ph.CO]].

15. S. di Serego Alighieri, Int. J. Mod. Phys. D 24 (2015) 06, 1530016 doi:10.1142/S0218271815300165 [arXiv:1501.06460 [astro-ph.CO]].

16. W. T. Ni, Rept. Prog. Phys. 73 (2010) 056901 doi:10.1088/0034-4885/73/5/056901 [arXiv:0912.5057 [gr-qc]]. 
17. A. Gruppuso, M. Gerbino, P. Natoli, L. Pagano, N. Mandolesi and D. Molinari, arXiv:1509.04157 [astro-ph.CO].

18. J. Kaufman, B. Keating and B. Johnson, arXiv:1409.8242 [astro-ph.CO].

19. C. R. Contaldi, arXiv:1510.02629 [astro-ph.CO].

20. C. Q. Geng, S. H. Ho and J. N. Ng, JCAP 0709 (2007) 010 doi:10.1088/14757516/2007/09/010 [arXiv:0706.0080 [astro-ph]].

21. G. Gubitosi, M. Martinelli and L. Pagano, JCAP 1412 (2014) 12, 020 doi:10.1088/1475-7516/2014/12/020 [arXiv:1410.1799 [astro-ph.CO]].

22. L. Pagano et al., Phys. Rev. D 80 (2009) 043522 doi:10.1103/PhysRevD.80.043522 [arXiv:0905.1651 [astro-ph.CO]].

23. G. Gubitosi and F. Paci, JCAP 1302 (2013) 020 doi:10.1088/1475-7516/2013/02/020 [arXiv:1211.3321 [astro-ph.CO]].

24. M. Galaverni, G. Gubitosi, F. Paci and F. Finelli, JCAP 1508 (2015) 08, 031 doi:10.1088/1475-7516/2015/08/031 [arXiv:1411.6287 [astro-ph.CO]].

25. G. Gubitosi, M. Migliaccio, L. Pagano, G. Amelino-Camelia, A. Melchiorri, P. Natoli and G. Polenta, JCAP 1111 (2011) 003 doi:10.1088/1475-7516/2011/11/003 [arXiv:1106.6049 [astro-ph.CO]].

26. V. Gluscevic, D. Hanson, M. Kamionkowski and C. M. Hirata, Phys. Rev. D 86 (2012) 103529 doi:10.1103/PhysRevD.86.103529 [arXiv:1206.5546 [astro-ph.CO]]. 
Table 1. Constraints on both the systematic error $(\beta)$ and the parity violation effect $\left(\alpha_{0}\right)$ using WMAP9 and BICEP1 datasets, together with constraints on the other cosmological parameters. All values are marginalized values and the errors are at $68 \%$ c.l.

\begin{tabular}{|c|c|c|}
\hline & $\beta$ & $\alpha_{0}$ \\
\hline Parameter & & \\
\hline$\Omega_{b} h^{2}$ & $0.0228 \pm 0.0005$ & $0.0228 \pm 0.0005$ \\
$\Omega_{c} h^{2}$ & $0.112 \pm 0.004$ & $0.112 \pm 0.004$ \\
$\theta$ & $1.040 \pm 0.002$ & $1.040 \pm 0.002$ \\
$\tau$ & $0.089 \pm 0.01$ & $0.089 \pm 0.01$ \\
$n_{s}$ & $0.973 \pm 0.01$ & $0.974 \pm 0.01$ \\
$\log \left(10^{10} A_{s}\right)$ & $3.17 \pm 0.04$ & $3.17 \pm 0.04$ \\
$\alpha_{0}$ & - & $-1.71 \pm 0.69$ \\
$\beta$ & $-1.77 \pm 0.71$ & - \\
$H_{0}$ & $70.9 \pm 2.1$ & $70.9 \pm 2.2$ \\
\hline
\end{tabular}

Table 2. Forecasted constraints on both the systematic error $(\beta)$ and the parity violation effect $\left(\alpha_{0}\right)$ using PLANCK and Polar-Bear mock datasets, together with constraints on the other cosmological parameters. All values are marginalized values and the errors are at $68 \%$ c.l. The fiducial model is the one of the best-fit WMAP+BICEP for the $\Lambda$ CMD with lensing and parity violation $\left(\alpha_{0}\right)$, second column of Table 1 . In the last column we also provide constraints obtained assuming no parity-violation nor systematic effects.

\begin{tabular}{|c|c|c|c|}
\hline & $\alpha_{0}$ & $\beta$ & no parity-violation-like effects \\
\hline Parameter & & & \\
\hline$\Omega_{b} h^{2}$ & $0.02279 \pm 0.00008$ & $0.02280 \pm 0.00008$ & $0.02242 \pm 0.00008$ \\
$\Omega_{c} h^{2}$ & $0.1123 \pm 0.0007$ & $0.1121 \pm 0.0007$ & $0.1192 \pm 0.0008$ \\
$\theta$ & $1.0400 \pm 0.0002$ & $1.040 \pm 0.0002$ & $1.040 \pm 0.0002$ \\
$\tau$ & $0.089 \pm 0.003$ & $0.892 \pm 0.003$ & $0.0947 \pm 0.004$ \\
$n_{s}$ & $0.974 \pm 0.002$ & $0.974 \pm 0.002$ & $0.961 \pm 0.002$ \\
$\log \left(10^{10} A_{s}\right)$ & $3.17 \pm 0.01$ & $3.17 \pm 0.01$ & $3.24 \pm 0.01$ \\
$\alpha_{0}$ & $-1.71 \pm 0.01$ & - & - \\
$\beta$ & - & $-1.74 \pm 0.01$ & - \\
$H_{0}$ & $70.8 \pm 0.3$ & $70.9 \pm 0.3$ & $67.8 \pm 0.3$ \\
\hline
\end{tabular}

Table 3. Marginalized best fit values and $68 \%$ c.l. errors on cosmological parameters using Planck+PolarBear simulated data (assumed fiducial cosmology obtained from second column of 1) analyzed with parity-violating effect $\left(\alpha_{0}\right)$, no effect and no effect but allowing a variation of the $A_{L}$ parameter. Third and fifth columns report the shift with respect to the varying $\alpha_{0}$ best fit in unity of $\sigma$, for the no effect and free $A_{L}$ cases respectively.

\begin{tabular}{|c|c|c|c|c|c|}
\hline & $\alpha_{0}$ rotation & no parity violation & $\Delta / \sigma$ & no parity violation $+A_{L}$ & $\Delta / \sigma$ \\
\hline Parameter & & & & & \\
\hline$\Omega_{b} h^{2}$ & $0.02279 \pm 0.00008$ & $0.02242 \pm 0.00008$ & 0.5 & $0.02278 \pm 0.00009$ & 0.1 \\
$\Omega_{c} h^{2}$ & $0.1123 \pm 0.0007$ & $0.1192 \pm 0.0008$ & 8.6 & $0.1136 \pm 0.0009$ & 1.4 \\
$\theta$ & $1.040 \pm 0.0002$ & $1.040 \pm 0.0002$ & 0.0 & $1.040 \pm 0.0002$ & 0.0 \\
$\tau$ & $0.089 \pm 0.003$ & $0.095 \pm 0.004$ & 1.5 & $0.088 \pm 0.003$ & 0.3 \\
$n_{s}$ & $0.974 \pm 0.002$ & $0.961 \pm 0.002$ & 6.5 & $0.970 \pm 0.002$ & 2.0 \\
$\log \left(10^{10} A_{s}\right)$ & $3.17 \pm 0.01$ & $3.24 \pm 0.01$ & 7.0 & $3.18 \pm 0.01$ & 1.0 \\
$\alpha_{0}$ & $-1.71 \pm 0.01$ & - & - & - & - \\
$A_{L}$ & - & - & - & $1.29 \pm 0.03$ & - \\
$H_{0}$ & $70.8 \pm 0.3$ & $67.8 \pm 0.3$ & 10 & $70.3 \pm 0.4$ & 1.3 \\
\hline
\end{tabular}


Table 4. $95 \%$ confidence level limits on $r$ for mock Planck dataset with fiducial WMAP5 best-fit parameters and mixing angle $\beta$ as specified in the table.

\begin{tabular}{|l|c|c|c|}
\hline Channel & mixing $\beta$ & $\mathrm{r}$ (including $\beta$ ) & $\mathrm{r}($ without $\beta$ ) \\
\hline Planck 70GHz & -1.0 & $<0.27$ & $<0.27$ \\
\hline Planck 100GHz & -1.0 & $<0.082$ & $<0.087$ \\
\hline Planck 143GHz & -1.0 & $<0.035$ & $<0.039$ \\
\hline Planck 217GHz & -1.0 & $<0.077$ & $<0.079$ \\
\hline EPIC & -0.5 & $<0.00071$ & $0.00086_{-0.00056}^{+0.00078}$ \\
\hline
\end{tabular}

Table 5. Some of the presently available constraints on parity-violation angle $\alpha$ from CMB. The references relative to these constraints can be found in. ${ }^{23}$ We report the experiment from which data were taken, the observational frequency channels, the estimated value of the parity-violation angle, together with its statistical and systematic uncertainty (if only one error is present, it includes both statistical and systematic uncertainties).

\begin{tabular}{|c|c|c|}
\hline Experiment & Energy channels $(\mathrm{GHz})$ & $\beta \pm$ stat $( \pm$ syst $)(\mathrm{deg})$ \\
\hline WMAP7 & $41+61+94$ & $-0.9 \pm 1.4 \pm 1.5$ \\
BOOM03 & 145 & $-4.3 \pm 4.1$ \\
QUAD & 100 & $-1.89 \pm 2.24 \pm 0.5$ \\
QUAD & 150 & $0.83 \pm 0.94 \pm 0.5$ \\
BICEP & $100+150$ & $-2.60 \pm 1.02 \pm 0.7$ \\
\hline
\end{tabular}

Table 6. Constraints on the energy-dependence parameters, together with their uncertainties and corresponding reduced $\chi^{2}$. On the left results obtained by including QUaD $150 \mathrm{GHz}$ channel in the analysis and on the right results obtained not including that channel.

\begin{tabular}{|c||c|c||c|c|}
\hline \multicolumn{1}{|c||}{} & \multicolumn{2}{c||}{ with QUaD $150 \mathrm{GHz}$} & \multicolumn{2}{c|}{ without QUaD $150 \mathrm{GHz}$} \\
\hline parameter & fit value \pm error & reduced $\chi^{2}$ & fit value \pm error & reduced $\chi^{2}$ \\
\hline$\beta_{0}[\mathrm{deg}]$ & $-0.93 \pm 0.70$ & 1.4 & $-2.22 \pm 0.92$ & 0.17 \\
$l\left[\mathrm{deg} \cdot \mathrm{GHz}^{-1}\right]$ & $-6.1 \cdot 10^{-3} \pm 5.3 \cdot 10^{-3}$ & 1.5 & $-1.99 \cdot 10^{-2} \pm 0.78 \cdot 10^{-2}$ & 0.03 \\
$q\left[\mathrm{deg} \cdot \mathrm{GHz}^{-2}\right]$ & $-3.6 \cdot 10^{-5} \pm 3.6 \cdot 10^{-5}$ & 1.6 & $-1.44 \cdot 10^{-4} \pm 0.57 \cdot 10^{-4}$ & 0.06 \\
$h\left[\mathrm{deg} \cdot \mathrm{GHz}^{2}\right]$ & $-4.2 \cdot 10^{3} \pm 4.2 \cdot 10^{3}$ & 1.6 & $-5.0 \cdot 10^{3} \pm 4.3 \cdot 10^{3}$ & 1.32 \\
\hline
\end{tabular}

